\title{
Estudiante y líder: Abraham Valdelomar y su experiencia universitaria (1906-1913)
}

\section{Student and Leader: Abraham Valdelomar and University Education (1906-1913)}

\section{Emilio Rosario Pacahuala}

Universidad Tecnológica del Perú, Lima, Perú

Contacto: C19979@utp.edu.pe

https://orcid.org/0000-0003-2421-548X

\section{RESUMEN}

Abraham Valdelomar es reconocido por la comunidad académica gracias a su importante producción intelectual. A la vez, es caracterizado como un personaje polémico de inicios del siglo $\mathrm{XX}$, debido a su estrambótico comportamiento y peculiar forma de vestir. Diversos estudios han analizado adecuadamente el contenido estético de sus cuentos y ensayos; sin embargo, muy pocos han investigado la actividad política de Valdelomar, la cual inició desde épocas universitarias. Fue alumno en la Escuela de Ingenieros y posteriormente de la Universidad Nacional Mayor de San Marcos, donde, si bien asistió a clases de forma irregular en ambas instituciones, esto no lo eximió de participar en eventos importantes, especialmente para la comunidad sanmarquina, como fue la constitución del Centro Universitario. El objetivo de la presente investigación es describir la experiencia de Abraham Valdelomar como estudiante de ingeniería, así como su accionar en San Marcos. Nos enfocamos en las elecciones estudiantiles para la Presidencia del Centro Universitario, donde Valdelomar no obtuvo el respaldo mayoritario por parte de sus compañeros. Así, develaremos que el autor de El Caballero Carmelo pierde las elecciones estudiantiles porque era catalogado como un mal estudiante, además de ser acusado por sus rivales como un aliado directo de Guillermo Billinghurst, quien era presidente del Perú (1912-1914). La universidad San Marcos, en ese entonces, tenía una gran presencia de militantes del Partido Civil, organización política opositora a Billinghurst. Para este trabajo utilizaremos el método histórico, el cual nos permitirá una contextualización más adecuada de las fuentes, periódicos y memorias.

Palabras clave: Literatura; Educación; Intelectuales; Universidad; Cultura; Abraham Valdelomar

\section{ABSTRAC'T}

Abraham Valdelomar is recognized by the academic community thanks to his important intellectua production. At the same time, he is characterized as a controversial character of the early twentieth century, due to his bizarre behavior and peculiar way of dressing. Several studies have adequately analyzed the aesthetic content of his stories and essays; however, very few have investigated Valdelomar's political activity, which began in his university days. He was a student at the School of Engineers and later at the Universidad Nacional Mayor de San Marcos, where, although he attended classes irregularly in both institutions, this did not exempt him from participating in important events, especially for the San Marcos community, such as the constitution of the University Center. The objective of this research is to describe Abraham Valdelomar's experience as an engineering student, as well as his actions in San Marcos. We focus on the student elections for the Presidency of the University Center, where Valdelomar did not obtain the majority support of his peers. Thus, we will reveal that the author of El Caballero Carmelo lost the student elections because he was labeled as a bad student, besides being accused by his rivals as a direct ally of Guillermo Billinghurst, who was president of Peru (1912-1914). San Marcos University, at that time, had a large presence of militants of the Civil Party, a political organization opposed to Billinghurst. For this work we will use the historical method, which will allow us a more adequate contextualization of the sources, newspapers and memoirs.

Keywords: Literature; Education; Intellectuals; University; Culture; Abraham Valdelomar 


\section{Introducción}

Uno de los literatos más importantes de inicios del siglo XX es Abraham Valdelomar, caracterizado no solo por su estrambótica personalidad o peculiar forma de vestir. Valdelomar realizó importantes aportes al mundo académico como: El Caballero Carmelo o El vuelo de los cóndores, por solo mencionar algunas de sus más importantes obras. Empero, su experiencia universitaria ha sido una fase de su vida poco profundizada por parte de estudiosos como Luis Alberto Sánchez o Fabio Xammar.

En el presente artículo desarrollaremos ese período en la vida de Valdelomar tanto como estudiante en la Escuela de Ingenieros, donde no obtuvo auspiciosos resultados académicos debido a la falta de vocación, así como su experiencia en San Marcos, donde participó indirectamente en la constitución del Centro Universitario. En este espacio estudiantil promovió el desarrollo de círculos de lectura, al igual que la ayuda a estudiantes con escasos recursos económicos. En una segunda parte abordaremos el proceso electoral en el que se involucró para convertirse en presidente del mencionado Centro Universitario. Es importante señalar que en ambos centros de estudio no culminó su carrera, pero ello no lo eximió de adquirir importantes conocimientos académicos y substanciales relaciones personales con otros académicos.

\section{La infancia}

El 12 de abril de 1888 nació Pedro Abraham Valdelomar Pinto. Fue bautizado a los pocos días en la parroquia de San Jerónimo (16 de abril), tradición que la mayoría de los niños peruanos, durante esa época, cumplía a los pocos meses de nacer. Gran parte de su infancia transcurrió en la ciudad de Pisco, lugar que tenía un encanto natural gracias a la "belleza serena y extraña que acrecentaba el mar" (Valdelomar, 2001a, p. 157).

Don Anfiloquio, su padre, necesitaba buscar los medios económicos para que subsistan su esposa e hijos, por lo que decidió vincularse al régimen de Andrés Avelino Cáceres con el fin de conseguir un empleo formal. Empero, el apoyo brindado al gobierno cacerista produjo a largo plazo serias consecuencias, pues el también conocido Brujo de los Andes fue defenestrado del poder político en 1895 a manos de
Nicolás de Piérola después de una sangrienta guerra civil. Entre las innumerables medidas promovidas por el régimen pierolista estuvo la reorganización de la aduana de Pisco, en donde se removió a los empleados allí existentes. En consecuencia, Anfiloquio Valdelomar, que allí laboraba, quedó relegado de la carrera administrativa pública, lo que provocó problemas económicos en su familia.

Era necesario conseguir el sustento económico que permitiera satisfacer las necesidades propias de la familia, como la alimentación y el vestido, especialmente por el bien de los hijos. Esta situación obligó a los Valdelomar a mudarse a Chincha Alta, donde había mayores oportunidades laborales. Dicho lugar ofreció no solo un empleo digno para don Anfiloquio, también facilitó que los primogénitos accedan a una educación básica (saber leer y escribir); sin embargo, la formación académica era limitada, no existían escuelas complementarias ni universidades en la región. Si se deseaba tener mayor formación educativa, se debía migrar hacia la capital del país, lo que finalmente se realizó.

Al llegar a la urbe limeña, Valdelomar se inscribió en el prestigioso colegio Nuestra Señora de Guadalupe, una institución en donde a inicios del siglo XX asistían mayoritariamente los hijos de la clase media. Sin embargo, la escuela fiscal no tenía los ingresos económicos suficientes para mejorar su infraestructura, constituida desde mediados de la centuria decimonónica, encontrándose en un estado totalmente calamitoso producto de la falta de apoyo estatal y de benefactores privados que ayudaran en su resurgimiento material. Así lo describe José Gálvez: "[...] tenía una fachada pobre, de corralón, casi siempre pintada al temple de claro celeste o de ocre vivo. La puerta era amplia y en el solariego zaguán estaba la portería" (1966, p. 41). Pero ello no impidió que conservara su prestigio como un centro de excelencia para la formación de la niñez y juventud nacional.

La falta de inversión por parte del gobierno central en desmedro de la educación fue denunciada años después. Mientras Valdelomar alcanzaba un gran prestigio como periodista se encargaría de evidenciar los retrasos en el pago de salarios a los maestros en 1915, demostrando el maltrato al que estaba sometido el personal involucrado en el sistema educativo nacional: 
[... en medio del desbarajuste de las finanzas y al atraso con que se hace el servicio económico de las dependencias del Estado, hay una situación creada por la desavenencia del fisco que tiene un aspecto odioso. Es la que atraviesan los profesores del Colegio de Guadalupe que sufren constante atraso en el pago de las quincenas. (Valdelomar, 2001b, p. 21)

Durante su etapa escolar, Abraham Valdelomar se convirtió en uno de los artífices claves para la fundación de la revista La Idea Guadalupana, donde los estudiantes publicaban poemas y pequeños cuentos. Esta actividad reflejaba el interés de nuestro personaje desde muy joven por organizar a las personas y promover espacios de actividad cultural. Desde 1901 a 1904 realizó sus estudios secundarios, tal como lo registra Luis Alberto Sánchez, quien lo calificó como un alumno promedio debido a que sus notas no eran sobresalientes, pero tampoco obtenía calificativos desaprobatorios (1966, pp. 8-9). Ello con excepción del último año, en donde se colige que de "las buenas calificaciones obtenidas por Abraham Valdelomar en los cursos de geometría, podría haberse extraído un pronóstico acertado, gusto por el arte" (De Priego, 2000, p. 53).

Como apreciamos, Abraham Valdelomar llegó a Lima desde Ica con el fin de mejorar sus condiciones de vida, para ello utilizó el móvil educativo como herramienta para alcanzar dichos fines.

\section{La educación universitaria}

La educación era el medio ideal para escalar socialmente, pues ya el propio sistema económico obligaba a profesionalizarse en alguna rama. Para alcanzar este objetivo se debía asistir a la universidad, egresar de la misma y así convertirse en miembro del sector profesional de nuestro país, debido a las exigencias de la sociedad por tener personas con algún tipo de especialización superior para servir al desarrollo nacional.

Finiquitada su experiencia colegial, Valdelomar decidió continuar estudios superiores, pero tenía dudas sobre qué universidad elegir. En un primer momento nuestro personaje optó por inscribirse como alumno en la Escuela de Ingenieros, actual Universidad Nacional de Ingeniería (Gonzales y Paredes, 2005, p. 36) donde tendría que pasar por la sección preparatoria:
[...] al comienzo, programados para un año, pero generalmente los alumnos necesitaban uno o dos años adicionales para concluirlos y quedar expeditos para el ingreso a las especialidades. Por eso, en 1905, un año antes de que ingresase Valdelomar, se extendieron los estudios preparatorios a dos años. Si el alumno no conseguía pasar satisfactoriamente todos los cursos, se le concedía un año adicional e, incluso, hasta dos años si el estudiante como será el caso de Valdelomar - por causas supuestamente excepcionales, no había logrado el nivel requerido para seguir las especialidades de ingeniería. (López Soria, 2007, p. 10)

Valdelomar asistió a la Escuela de Ingenieros durante cinco años, no superando la sección inicial o los estudios formativos de Ciencias y Letras. Los problemas económicos fueron un factor determinante que le impidieron culminar sus estudios universitarios, lo que derivó en la obligación de asumir diversas actividades laborales que permitieran satisfacer sus necesidades básicas, relegando las responsabilidades universitarias a un segundo plano.

Esta situación no le impidió, sin embargo, seguir buscando obtener una carrera profesional. Gracias a la ayuda de Luis Varela y Orbegoso - conocido en el mundo periodístico como Clovis y que se convirtió en una influencia importante para introducirlo en el mundo del periodismo años después - se inclinó por iniciar sus estudios en otra especialidad. Así, ingresó a la Facultad de Letras de la Universidad de San Marcos, la cual tenía su propia dinámica académica. Por ejemplo, hoy en día el estudiante universitario ingresa directamente a la profesión que elige después de sortear exitosamente el concurso de admisión y adquirir una de las vacantes en disputa. En los tiempos de Valdelomar, todos los estudiantes elegían alguna profesión al interior de los claustros universitarios; quiere decir, después de asistir a dos años de formación general, los otros años posteriores los alumnos desarrollaban estudios de especialización, sea en jurisprudencia, medicina o teología, por citar algunas de las carreras profesionales ofrecidas por la Decana de América.

Un aspecto que debemos resaltar es el incremento del número de matrículas en la Universidad de San Marcos durante los primeros años del siglo XX: 


\section{Año Población universitaria Matriculados en San Marcos}

$\begin{array}{lll}1902 & 1307 & 976 \\ 1907 & 1160 & 789 \\ 1912 & 1667 & 1164 \\ 1917 & 1985 & 1331\end{array}$

Fuente: Deustua y Rénique, 1989, p. 20.

Estos cambios al interior de la Universidad de San Marcos fueron analizados por Marcos Garfias (2009), quien señala que el exponencial ascenso de las matrículas universitarias respondía a la demanda que exigía el país por contar con una importante cantidad de profesionales; ello con la finalidad de edificar fábricas, mantener las vías de comunicación, impulsar la extracción de minerales, entre otros rubros de vital importancia para la economía de la nación.

Estas características nos permiten entender el universoeducativo delas primeras décadas delsigloXX, cuyos cambios reconfiguraron la sociedad peruana; un escenario donde Valdelomar se desenvolvió como estudiante universitario sanmarquino.

\section{La universidad peruana}

San Marcos, durante la etapa republicana, se constituyó en el centro educativo universitario por excelencia; el espacio académico en donde se forjaba exclusivamente la élite política e intelectual del país.

$\mathrm{Al}$ ingresar a los claustros sanmarquinos, Valdelomar percibió la tensa relación entre los docentes (amos y señores de las cátedras) y los estudiantes; especialmente aquellos que pertenecían a la clase media, cuyo número crecía considerablemente debido a la promesa de ascenso social producto del desarrollo del conocimiento:

[...] sin embargo, San Marcos empezó a cambiar: la primera oleada de estudiantes provincianos fue de gente acaudalada, con lo que la conformación social no se vio radicalmente afectada. Pero durante la segunda oleada, intensificada en los años 10 , ingresaría un fuerte componente de clase media. En una década esta universidad adquirió un sustrato social más variado y empezó a politizarse con intención de actuar fuera de su espacio, en los ámbitos más públicos. (Del Águila, 1997, p. 53)

La presencia de estudiantes de diversas partes del país debía obligar al cuerpo docente a utilizar otras herramientas para transferir la información con éxito; por ejemplo, utilizar casos prácticos, investigaciones dirigidas a los trabajos de campo, un análisis sobre la heterogeneidad de la población, pero ninguna de estas estrategias pedagógicas llegó a ejecutarse. Los profesores de San Marcos, en los albores del siglo $\mathrm{XX}$, tenían una pedagogía y estrategia de enseñanza destinada a un tipo de estudiante pasivo que solo escuchara, recepcionara y no debatiera las ideas del docente. Un alumno que memorizara al pie de la letra las lecciones vertidas en clases.

La enseñanza a inicios del siglo pasado demandaba que los contenidos de las diferentes materias dialoguen con la realidad, que tengan mayores ejemplos para su comprensión; por ende, tendría que modificarse la ideología educativa si se quería constituir profesionales que exploten racionalmente los recursos de todo el territorio patrio, propongan medidas jurídicas que mantengan el orden social del país y, lo más importante, ejecuten los cambios estructurales en beneficio de toda la comunidad peruana. Al no satisfacer la demanda estudiantil, la cual clamaba una forma distinta de enseñar, surgió una oposición en contra del manejo de la universidad. Como los maestros no eran permutados de sus cátedras para ceder el paso a docentes que impartan el conocimiento que se necesitaba y la transferencia del conocimiento adecuado, las masas estudiantiles reaccionaron promoviendo la tacha de los llamados "malos profesores". La efectivización se realizó desde 1918, gracias a la reforma universitaria por la cual los estudiantes obtuvieron un mayor protagonismo en el gobierno universitario y pasaron a formar parte de las reformas propuestas.

Una alternativa para combatir la mediocre enseñanza universitaria era la promoción de actividades extracurriculares, como los círculos de debate o grupos de lectura, opciones que nuestro personaje promovió al interior de San Marcos. El objetivo era alimentar el entusiasmo académico por parte del alumnado; sin embargo, los estudiantes universitarios no solo 
concentraron su interés en el desarrollo académico y administrativo, también tomaron la decisión de involucrarse en la vida política de su país y canalizar las demandas sociales de la época.

Para permutar esa realidad en la que estaba envuelta la universidad más importante del país, Valdelomar ingresó a participar en la vida política universitaria. De esta manera, junto con sus compañeros, apoyó la formación del Centro Universitario, el primer órgano estudiantil cuyo fin original fue mejorar la calidad educativa, reforzar la enseñanza de las materias académicas e incluso apoyar materialmente a los estudiantes para lograr un sobresaliente desarrollo intelectual. Empero, lo que en un comienzo fue un espacio de apoyo educativo en favor de los alumnos con escasos recursos económicos, con el paso de los meses se convirtió en un lugar de disputa política por su control; ello debido a que dicha agremiación estudiantil tuvo una ingente capacidad de influencia entre el alumnado, dado los diversos servicios académicos y sociales que ofrecía. La agrupación que deseaba mantener el control estaba compuesta por los militantes del Partido Civil. junta directiva el testimonio donde se relata que la edificación del Centro Universitario fue un trabajo conjunto por parte de los estudiantes:

[...] la idea de un Centro Universitario nació como un ensueño, cristalizado como una necesidad; todos los cerebros lo pensaron y todos los corazones lo sintieron [...] larga y fatigosa ha sido la tarea. Difícil es para los jóvenes aptos para soñar y para sentir, aprender a organizar; y hay, vencidos los obstáculos, a [...] la jornada, volvemos los ojos al punto de partida, para exponer nuestros trabajos y mostrarnos con el recuerdo, el sendero que abrimos con la voluntad. (Memorias, 1908, p. 2)

En la inauguración del Centro Universitario participó el presidente de la República José Pardo y Barreda, por lo que observamos lo importante que era la Universidad de San Marcos en la vida del país. El motivo fundamental del porqué el interés de los mandatarios nacionales en generar una política de buena vecindad con los sanmarquinos se debe a que ellos eran los destinados a integrar la comunidad intelectual del país; además, sus egresados se

\section{Una alternativa para combatir la mediocre enseñanza universitaria era la promoción de activi-} dades extracurriculares, como los círculos de debate o grupos de lectura, opciones que nuestro personaje promovió al interior de San Marcos. El objetivo era alimentar el entusiasmo académico por parte del alumnado; sin embargo, los estudiantes universitarios no solo concentraron su interés en el desarrollo académico y administrativo, también tomaron la decisión de involucrarse en la vida política de su país y canalizar las demandas sociales de la época

Recordemos que el civilismo tenía un vasto control de la burocracia y el profesorado de San Marcos. Una muestra del dominio en el aparato administrativo sanmarquino por parte del Partido Civil son las repetidas elecciones de José Pardo y Barreda como rector de esta casa de estudios o su presencia como decano de la facultad de Jurisprudencia, además de la participación de distintos catedráticos sanmarquinos en los diversos ministerios del aparato estatal.

La edificación del Centro Universitario se convirtió en la primera victoria obtenida por el alumnado al establecer un lugar en favor de la clase media, por tanto "el centro ha triunfado por ser la obra soñada por toda nuestra juventud..." (Valdelomar, 2001c, p. 120). Para afianzar esta versión encontramos en las memorias de la primera convertirían en el sector capacitado para desarrollar económica, política y socialmente a la nación. Así mismo, San Marcos se ubicaba estratégicamente cerca del circuito de poder nacional: el Parlamento Nacional y el Palacio de Gobierno.

Los alumnos gozaron de las flamantes instalaciones del Centro Universitario, utilizando incluso dicho lugar como un espacio de distracción y diversión, además de las funciones ya mencionadas:

[...] las noches generalmente son dedicadas al centro universitario donde se hace brillar, música y sport. Hay en el local del centro una sencilla sala de gimnasia, dos mesas de billar, una linda biblioteca y un bar bien provisto, que los domingos en los meses de poco estudio, 
prepara almuerzos a los que concurren grupos de universitarios. (Valdelomar, 2001d, p. 133)

Este tipo de actividades respondía a fortalecer los vínculos de hermandad y amistad al interior del cuerpo estudiantil y entre los diversos sectores sociales, tal cual eran los objetivos primarios del Centro Universitario:

[...] nuestro principal objeto es conseguir la unión de los estudiantes, creando vínculos de solidaridad y de afecto, procurando hacerlos comunes en los fines, hermanos en los ideales, lograr por medio del trato personal y continuo la vigorización del organismo colectivo noble en su tendencia y fuerte en su acción; combatir los inútiles egoísmos creados por la diferencia de estudios, integrando en lo posible las mentalidades y constituirnos, nosotros mismos, en consejo y auxilio, interviniendo en la acción universitaria y social de los asociados. (Memorias, 1908, p. 3)

Además, otro de sus objetivos fundacionales era ayudar económicamente a los estudiantes con menos recursos para que continúen asistiendo regularmente a clases:

[...] la razón por la cual se dan tan pocas conferencias al año se debe a que la extensión universitaria está encomendada a un pequeño grupo de jóvenes, que si bien están dotados de excepcionales condiciones para la obra, su obra no es tan amplia como lo sería si todos los universitarios sin excepción ninguna, fueran colaboradores en esta noble y democrática labor. (Morales, 1912, p. 3)

Esta política asistencialista en favor de los "desfavorecidos" no era nueva en nuestro país. Desde el siglo XIX, las élites impulsaron la creación de la Beneficencia de Lima como institución de auxilio para los más desfavorecidos. Pero los integrantes del Centro Universitario fueron mucho más allá de establecer una remuneración económica en beneficio de los estudiantes para que cubriesen sus necesidades básicas, buscaron incluso brindar un apoyo íntegro; ello se denota en el servicio médico en favor de sus compañeros, razón de su éxito en convertirse en una institución de fuerte influencia al interior de la comunidad estudiantil:
[...] muchos estudiantes viven en Lima, separados de sus familias y en caso de enfermedad no tienen la mano tierna de los suyos que acaricie y asista. Fuerza era preocuparse de subsanar en alguna forma ese desamparo y nadie con más deber ni con más derecho que sus compañeros los estudiantes, hermanos por el ideal y por el afecto. (Memorias, 1908, p. 4)

Entre los firmantes del primer manifiesto se encontraban José Gálvez, Carlos Monge, José Antonio de Lavalle, Alberto Alexander, Alberto Martín Linch, Edilberto Noza, Hermilio Valdizán, César Patrón, Aníbal Solano, Fernando Tola, Juan de Cárdenas y Oscar Miró Quesada.

Un año después, la asamblea general de estudiantes ratificó los acuerdos iniciales que aportaron en la fundación del Centro Universitario, pero adicionando dos elementos importantes que nos ayudarán a entender la transformación interna de San Marcos. Primero, se promovería el "acercamiento de maestros y estudiantes" mediante la liquidación de esa radical relación vertical entre ambos estamentos universitarios para lograr una impartición de la enseñanza más fructífera, y donde respetuosamente se cuestionen las ideas de los maestros, lo que coadyuvaría a un diálogo profundo sobre los problemas de la nación. Lo segundo era "indicar la ejecución de labores de orden social que convenga llevar a cabo", en otras palabras, forjar una política de apoyo hacia el estudiante por parte de la propia universidad, lo cual garantice mejores condiciones para el desarrollo académico.

El Centro Universitario tuvo una importante propuesta: la publicación de una revista. Esta tendría como fin no solo promocionar sus acuerdos y manifiestos en favor de los estudiantes o desarrollar las actividades sociales para la comunidad, sino también era una oportunidad para que los alumnos difundan sus investigaciones, opiniones y aportes al desarrollo económico, político y social de la nación. El primer comité editorial fue integrado por Julio C. Tello, Alfredo González Prada, Percy Gibson y Pablo Abril de Vivero. Una interrogante que nace naturalmente es por qué Valdelomar no participó activamente en este espacio académico tal cual lo hizo en la revista La Idea Guadalupana, realizada en su etapa escolar. Su experiencia adquirida hubiese sido fructífera en favor de la publicación universitaria. Una de las respuestas 


\section{Valdelomar no encontró el apoyo por parte de la población estudiantil. La causa de la falta de atracción tuvo su origen en que sus rivales de turno señalaron (...) que no era un estudiante modelo, menos aún un ejemplo a seguir por la juventud universitaria. Esta fama fue atribuida a las constantes matrículas en los mismos cursos, sus notas desaprobatorias, sus mediocres participaciones en clase e incluso su vinculación con el gobierno de Billinghurst, el cual no mantuvo una gran popularidad entre la población}

se debe a su falta de diplomacia para establecer buenas relaciones con sus compañeros generacionales. $\mathrm{Su}$ personalidad, en un primer momento, se convirtió en un gran escollo que dificultaba constituir un grupo de personas que ayude a gestar algún proyecto al interior de la universidad desde esta etapa; él tuvo que comprender que para forjar una relación interpersonal estable se debía ceder y comprender las múltiples personalidades, aceptar las diferencias de opiniones e incluso mantener una relación cordial a pesar de hondas discrepancias ideológicas. Pero tampoco Valdelomar fue un paria social, de manera astuta constituyó una importante red mínima de amistades estratégicas que más adelante le ayudarían en el desarrollo de su vida política y profesional; tal es el caso, por ejemplo, de José de la Riva-Agüero y Osma, quien formó parte de la comunidad sanmarquina, paradójicamente un miembro de la élite con quien debía tener una relación confrontacional, pero no fue así.

Otro de los personajes relacionados con el autor de El Caballero Carmelo desde épocas universitarias fue el escritor Alfredo González Prada (hijo del ensayista Manuel González Prada), quien incluso se doctoró en Letras. Abraham Valdelomar no tuvo la misma suerte; él truncó sus estudios debido a diversos factores, por ejemplo: los pésimos resultados académicos, los cuales generaban que lleve las materias en repetidas oportunidades, la falta de capacidad económica para solventar sus necesidades básicas e incluso sus malas relaciones con los miembros del Centro Universitario evitaron que tuviera el apoyo necesario para llevar exitosamente una vida universitaria.

Sin embargo, durante su estadía en San Marcos se estableció un momento culminante que reforzó su decisión de incursionar en el mundo de la política nacional. Él y sus compañeros decidieron realizar una excursión a la sierra sureña; esta fue la oportunidad para reencontrarse con ese Perú Profundo, no la provincia costera que representaba Ica (cuasi modernizada). Nos referimos a esos espacios geográficos tan explotados y marginados por los españoles desde épocas coloniales y por los mistis durante la República; pero Valdelomar no fue el pionero en torno a denunciar los males a los que estaban sometidas las poblaciones de la sierra. Los indigenistas Pedro Zulen o Dora Mayer realizaron una titánica labor a través de panfletos y diarios, en donde exhibían el abuso hacia el indígena por parte del hombre "blanco". Pero no solo él, todos los estudiantes universitarios fueron testigos del abandono que sufrían los pobladores del Ande, quienes no tenían los suficientes derechos políticos que permitan ser reconocidos como integrantes de la comunidad nacional; además, escucharon de los abusos a los que estaban sometidos, como por ejemplo el despojo de sus tierras.

El primer lugar visitado fue Arequipa. La emoción de los universitarios y del propio Valdelomar fue descrita en un conjunto de crónicas publicadas en el diario El Comercio, en donde nuestro personaje asumió la tarea de corresponsal. En las crónicas describió los pormenores de las edificaciones citadinas y las costumbres locales, además concentró su interés en una de las maravillas naturales más importante de dicho lugar: "El Misti repiten todos y se precipitan a las ventanas y a los balcones del carro. Es el Misti, en efecto. El Misti soñado tantas veces, imaginado otras tantas". La atención brindada por parte de la población fue de total amabilidad, tal como lo testimonia Valdelomar.

La visita de la representación sanmarquina culminó con un agasajo culinario por parte de las autoridades de la ciudad, quienes nombraron a los jóvenes estudiantes como ilustres invitados. El porqué de tanta atención a los alumnos tiene dos explicaciones; en primer lugar, el prestigio de una universidad limeña como San Marcos generaba asombro en los ciudadanos de las provincias, quienes señalaban que el ingresar a participar en la élite académica capitalina era todo un éxito profesional dado que Lima monopolizaba la impartición del conocimiento. En segundo lugar, porque ellos se convertían en dignos 
representantes de la modernidad, ejemplos a seguir por el resto de la comunidad nacional, denotándose en el habla, en la forma de vestir, en las profesiones que desempeñarían, entre otros indicadores que los representaba como ciudadanos modelos.

Esta situación de rendir agasajo a los jóvenes visitantes también se repitió en Puno, lo que nos permite entender que el ser universitario y sanmarquino - en aquellos tiempos - provocaba un fuerte prestigio en la sociedad a escala nacional. Recordemos que existían pocos centros de educación superior, por tanto, el valor simbólico de un estudiante universitario era ingente. El deleite personal, al sentirse admirado, era un privilegio el cual nuestro personaje no estaba dispuesto a perderlo; por ello, buscó todos los medios para seguir perteneciendo a la comunidad universitaria y gozar de los beneficios sociales que de ella obtenía. Más adelante, Valdelomar intentó volver a empoderarse al interior de la Decana de América al aspirar convertirse en presidente del Centro Universitario.

No obstante, la situación económica lo obligó a abandonar los estudios universitarios. Valdelomar decidió realizar una nueva acción: participar en la política nacional al apoyar a Guillermo Billinghurst, quien se convirtió en presidente de la República en 1912. Esta situación conllevó a asumir importantes cargos burocráticos, como la dirección del diario oficial El Peruano; empero, decidió no conformarse en ser un burócrata estatal, su nuevo objetivo era reivindicarse al interior de la universidad peruana. Pero su finalidad no era solo culminar sus estudios, ahora buscaba convertirse en presidente del Centro Universitario.

\section{Elecciones estudiantiles}

En 1913 Abraham Valdelomar postuló a la presidencia del Centro Universitario, organización que nació con un objetivo principal: el ayudar a los estudiantes con menos recursos económicos a realizar exitosamente su vida académica. La junta directiva original fue conformada por "jóvenes católicos que presidía Rey Boza y del cual eran miembros Federico Panizo y José María de la Jara" (Belaunde, 1967, p. 267); ellos se encontraban estrechamente vinculados al Partido Civil.

De esta manera, los civilistas ostentaron el control del gremio estudiantil desde su fundación; gracias a ello tuvieron una amplia influencia en el estudiantado ya que suplían sus carencias básicas: alimentación y vestido. Nuestro personaje buscó arrebatarles dicho espacio estudiantil. Esta aspiración por parte de Valdelomar estuvo alentada por el incremento de la clase media al interior de la universidad Decana de América, lo cual parecía convertirse en una ventaja a su favor, ya que dichos votantes apoyarían "naturalmente" su elección.

Si bien Valdelomar había pasado por una experiencia electoral de mayor envergadura como eran los comicios presidenciales de 1912, parecería extraño el optar por un espacio universitario de aparentemente menor importancia. Sin embargo, la influencia en San Marcos era vital para cualquier gobierno, debido a que constituyó uno de los más importantes centros de estudios de América Latina, además de convertirse en una de las instituciones de vital apoyo para el civilismo debido a que muchos de sus más connotados miembros eran catedráticos o autoridades de dicha institución educativa:

[p]ara la historiografía peruana que se ha ocupado de este período, la Universidad de San Marcos fue una institución funcional a la oligarquía civilista en el poder porque además de ser un espacio de tránsito natural en la instrucción de los hijos de la elite estaba controlado por el Partido Civilista. Gran parte de los catedráticos y las autoridades universitarias eran miembros del partido y muchos de ellos compartían sus labores docentes y administrativas con la actividad política y gubernamental. (Garfias, 2009, p. 149)

Por lo tanto, Valdelomar, como parte aliada del gobierno, necesitaba recuperar un espacio que había nacido para empoderar a los estudiantes y apoyarlos en su desarrollo académico. No obstante, el Centro Universitario, debido a los servicios que ofrecía en esos momentos al brindar alimento y apoyo académico, se convertiría en un espacio de reproducción de fidelidades favorables al civilismo.

Los acontecimientos acaecidos en San Marcos concertaban el interés público e incluso la prensa nacional dedicaba parte de su cobertura informativa para narrar los diversos eventos y también losproblemas institucionales al interior de esta casa de estudios. Por ejemplo, el 9 de abril de 1913 se informaba sobre 
el posible cierre de la Escuela de Agricultura, dicha medida sería discutida en el Centro Universitario por los estudiantes. Los acuerdos de esa asamblea serían elevados a las autoridades universitarias para que sean atendidos; así mismo, se informaba sobre la elección de representantes estudiantiles en las distintas facultades, los cuales elevarían sus demandas a los decanos y al mismo rector.

Para inicios de 1910, los estudiantes no tenían una organización que canalizara sus demandas académicas o lo relacionado con los servicios sociales, tal como lo señalaba $\mathrm{El}$ Comercio: "hasta ahora ninguna manifestación colectiva se ha solidarizado con el espíritu de la juventud ante cualquier problema que la atañe directamente". Esta orfandad fue detectada por Valdelomar, quien vislumbró un terreno fértil para organizar a los alumnos y con ello obtener un protagonismo al interior del corpus estudiantil. No es extraño, por lo tanto, que nuestro personaje forjara los batallones universitarios que respaldaron la elección de Billinghurst en 1912, quien finalmente obtendría la Presidencia de la República. Con esta exitosa experiencia, un año después (1913) buscaba empoderarse como presidente del Centro Universitario.

La candidatura de Valdelomar provocó, desde un primer momento, bastante resistencia entre los estudiantes universitarios. Fiel a un estilo que lo caracterizaría, su presencia debía marcar un interés público y de forma pomposa; así, nuestro personaje decidió presentarse oficialmente como aspirante a la representación estudiantil del Centro Universitario en un evento público, organizando un almuerzo de confraternidad realizado en el comedor de la mentada institución. Entre los principales invitados a dicho evento se encontraban: Edgardo Rebagliati, Federico Villarreal y otros destacados hombres que más adelante se convertirán en influyentes académicos peruanos. Sin embargo, esta reunión fue criticada virulentamente por sus opositores, señalando que no convocó una masiva concurrencia por parte del alumnado sanmarquino; por el contrario, solo tuvo una baja participación estudiantil, revelando que su imagen estaba "cimentada sobre tan enorme y decantada popularidad". Esta denuncia fue hecha por Víctor E. Gómez Sánchez, quien alegaba que el factor principal de rechazo por parte de la comunidad sanmarquina se debía a los pésimos resultados académicos obtenidos por Abraham Valdelomar mientras cursaba los estudios generales:

[...] por qué no tengo opción a la presidencia del Centro Universitario, pues no es justo que una persona que no tiene - sea porque no quiere o cualquier otro motivo- un solo curso universitario aprobado, vaya a ocupar el primer lugar entre todos los estudiantes peruanos.

La respuesta a este tipo de manifestaciones fue confrontada por Rebagliati, quien desmintió que este almuerzo haya sido parte de una estrategia proselitista. La presencia de Abraham Valdelomar se debió a un agasajo para celebrar su onomástico, por tanto, su intencionalidad no era de carácter electoral. Más aún este evento tuvo la participación de más de 180 universitarios, siendo señal de que una importante cantidad de alumnos admiraba y respetaba a nuestro personaje. Pero Gómez Sánchez no fue la única persona que públicamente expresó su inconformidad en contra de la candidatura de Valdelomar. Otro estudiante universitario de nombre Federico Copiona señalaba que era "un elemento nocivo y pernicioso" en caso de asumir la presidencia del Centro Universitario, debido a su cuestionable devenir académico y cercanía a un gobierno sin legitimación alguna.

Esta situación generó que se forje un bando opositor entre aquellos que no podían permitir que un hombre con una cuestionada reputación asuma el poder y representatividad de una institución académica. El rival de Abraham Valdelomar inicialmente sería Hernán C. Bellido, pero él decidió declinar su candidatura en favor de Fernando Tola, de enorme prestigio en el universo estudiantil, gracias a sus relevantes méritos por el brillo de su actuación como "leader" de la delegación del Perú en el congreso de estudiantes desarrollado en Uruguay; y, lo más importante, porque era el personaje lo suficientemente conciliador entre todos los estamentos que conformaban el universo sanmarquino. Empero, la causa real del porqué Bellido se retiró de la contienda electoral se debió a que no se sentía capaz de derrotar a Valdelomar, quien tendría ventajas como representante del gobierno central (desde promesas de empleos hasta amedrentamiento físico a sus rivales); se reproducían, así, las estrategias que utilizó el civilismo para mantener su domino durante gran parte de la República Aristocrática, pero no sería la única arma. 
El discurso valdelomarista, conforme se acercaba el momento de llevar acabo las elecciones, se radicalizó a tal punto que buscaba provocar un escenario confrontacional con sus rivales vinculados al civilismo. Esta situación debía favorecer al autor del Vuelo de los cóndores, debido a que la mesocracia conformaba la mayoría de la población universitaria. Este escenario fue denunciado por Hernán Bellido, quien en una carta abierta publicada en el diario La Prensa criticó este proceso de polarización que el discurso valdelomariano intentaba formular al interior de San Marcos:

[...] los métodos empleados para conseguir adherentes por los patrocinadores de otra candidatura, cuyo carácter universitario ha sido y es discutido, ya que las animaciones de sus mismos adictos la revisten de un aspecto político evidente, han determinado en mí la resolución que origina esta carta. Y no puede ser de otra manera: sojuzgar voluntades por medio del ofrecimiento de puestos públicos: amenazar con la destitución a quienes en ejercicio de alguno no simpatizan con esta candidatura; explotar el sentimiento regionalista atribuyendo a los estudiantes limeños a un necio afán de despolitizar a los de provincias; afirmar en todos los tonos que, en caso de un fracaso para la candidatura del centro universitario, no sólo no encontraría apoyo sino hostilidad de parte el gobierno: conseguir que se ejerciten influencias personales cuyas vinculaciones obligan a muchos; introducir en el proceso electoral universitario los sistemas que vician los procesos políticos, maleando así el ambiente moral de nuestros claustros y conseguir votos por los mismos medios que se estilan en aquellas contiendas; no pueden dejar de producir en el espíritu de quienes siempre hemos batallado por mantener incólumes los ideales universitarios de independencia, sinceridad y rectitud, una dolorosa impresión.

La respuesta de Valdelomar fue calificar a Hernán Bellido de realizar afirmaciones temerarias sin evidencia alguna, negando en todo momento la utilización de métodos clientelísticos o amenazas a los universitarios; alegó que su estilo siempre ha sido el promover el debate alturado y el respeto a las opiniones contrarias, tal cual señalan los preceptos modernos de la democracia (Valdelomar, 2001e). Por el contrario, Abraham Valdelomar denunció la actitud de Bellido, quien junto con sus amigos representaron un obstáculo para el buen desempeño de las juntas directivas anteriores:

[...] ahora que el señor Bellido ha desistido de su candidatura, por carta publicada en los diarios, debo manifestar a ud lo siguiente, que es falso, absolutamente falso y calumnioso, que yo o mis amigos hayamos presionado a alguien con dádivas u ofrecimientos, aseveración que envuelve un grosero insulto a todos los universitarios a quienes se cree capaces de tal villanía; que la campaña contra mí ha sido hecha por un conocido círculo de jóvenes, como el señor Ulloa, que tomaron como escudo al señor Bellido y que no es sino la continuación de la que el mismo círculo emprendió contra el señor Dulanto, actual presidente del Centro, esterilizando, como les consta a todos las iniciativas de este caballero, que el señor Bellido, que no tiene cómo justificar su derrota, ha redicho los términos pocos serios de su carta, por la falsedad que envuelven, debe extrañarse en quien pretendió ser presidente del Centro, y por fin invito a que se me pruebe los cargos de esta carta.

Después de tantas confrontaciones realizadas a través de la prensa escrita y álgidos debates acontecidos en los distintos pasillos universitarios, se llevó a cabo el proceso electoral. Gómez Sánchez y Seoane firmaron un pronunciamiento en La Crónica donde denunciaban que los seguidores de Valdelomar, de forma soterrada, anunciaban a sus amigos más cercanos a que asistan a las elecciones para ejercer su voto, sin tener la amabilidad de convocar a todos sus compañeros sin discriminación alguna para expresar libremente su opción. El objetivo con esta acción era asegurar las votaciones en favor de Abraham Valdelomar y evitar que los opositores puedan expresar electoralmente su rechazo.

Esta situación fortaleció al bando opositor. Así, los universitarios comenzaron a organizarse en contra de la candidatura de Abraham Valdelomar. Un ejemplo de ello son los estudiantes chalacos, quienes se comportaron reacios en aceptar a Valdelomar como el futuro presidente del Centro Universitario; el argumento de esta decisión respondía a que no era un estudiante modelo, por tanto no era digno de representarlos. Pero los ataques no solo se limitaron a la figura del candidato Valdelomar, sus seguidores también fueron criticados por sus repudiables actos: "nos 
limitamos hoy a declarar que es un pequeño círculo de elementos morbosos que rodea a un candidato impopular a la presidencia del Centro Universitario, el generador de todos estos actos vituperables", dicho pronunciamiento estuvo amparado por la firma de "los verdaderos universitarios".

A pesar de su contundente respuesta no solo por medios periodísticos, también de forma oral, Abraham Valdelomar no encontró el apoyo por parte de la población estudiantil. La causa de la falta de atracción tuvo su origen en que sus rivales de turno señalaron - como mencionamos en el párrafo anterior - que no era un estudiante modelo, menos aún un ejemplo a seguir por la juventud universitaria. Esta fama fue atribuida a las constantes matrículas en los mismos cursos, sus notas desaprobatorias, sus mediocres participaciones en clase e incluso su vinculación con el gobierno de Billinghurst, el cual no mantuvo una gran popularidad entre la población.

Tal situación de constantes acusaciones entre las diversas facciones generó que el ambiente sanmarquino fuese altamente confrontacional. El escritor Clemente Palma describe el escenario de violencia al interior de la universidad, promovido por los seguidores valdelomaristas durante los comicios electorales universitarios: "[...] en los claustros de la facultad de letras ha habido garrotazos y escándalos, los jóvenes van armados con revólveres y ha habido día en que no han podido funcionar las clases".

Estas actitudes responden a que nuestro personaje intentó reproducir las mismas estrategias que permitieron la victoria de 1912: el boicot, la presión popular y el enfrentamiento físico. Mas en el universo sanmarquino no tuvo un efecto positivo, no eran las calles limeñas y de provincias en donde era "justificable" este tipo de accionar; era un lugar de formación académica donde existían determinadas normas y comportamientos que debían respetarse y la institución debía ser resguardada por sus miembros.

La importancia de San Marcos y estas elecciones estuvieron retratadas en las páginas de los principales diarios de la época. Alberto Ulloa escribió una serie de artículos donde señalaba su férrea oposición en contra del discurso valdelomarista, el cual — según el cronista - representaba el vil oportunismo y la falta de honorabilidad por parte de quien aspiraba a representar a todos los estudiantes:
[...] desde que trascendió al público la noticia de que el señor Abraham Valdelomar lanzaba su candidatura a la presidencia del centro universitario, cuantos conservan en el espíritu la ilusión de una regeneración juvenil, se preguntaron con inquietud si no habría hoy ya, en las aulas carolinas, quienes tuvieran la energía bastante para levantar frente a ella, la muralla de una oposición decidida, vigorosa y sincera. (en Valdelomar, 2001e, p. 54)

Esta editorial provocó la ira de Valdelomar, cuyo honor y reputación fueron cuestionados leoninamente. Ante la falta de un discurso efectivista para responder los ataques de sus opositores, y en especial de Ulloa, decidió retarlo a un duelo para el 13 de mayo de 1913 por la publicación de dicho artículo que consideró difamatorio. Finalmente el duelo fue desarrollado sin causar la muerte de ninguno de los rivales; sin embargo, esta acción fue la estocada final para sepultar la candidatura de Abraham Valdelomar debido a que legitimaba el discurso violentista con el que fue calificado por sus rivales: un candidato sin alternativas para una mejora en el desarrollo académico e institucional de San Marcos, que solo tendría como herramientas el amedrentamiento y la amenaza, actos que fueron denunciados en sus caricaturas años atrás.

Finalmente, Abraham Valdelomar fue derrotado por Fernando Tola. La versión del propio personaje nuestro sobre las causas oficiales de la derrota radicaba en que los intereses subalternos evitaron que se encumbrara en el Centro Universitario, pero esta polarización en la que estuvo envuelta la universidad debía ser superada para gestar la unidad entre los estudiantes, ya que los ideales trascienden una elección. Este discurso conciliatorio era ideal para no sepultar totalmente su imagen, tan cuestionada al interior de San Marcos; así mismo, era la posición más estratégica para demostrar el ideal democrático de una persona, el respetar los acuerdos de la mayoría.

La derrota provocó en Valdelomar un profundo resentimiento, el cual quedó reflejado en una carta enviada a Bustamante y Ballivián redactada un año después del proceso electoral (Ángeles Caballero, 2007, p. 136). En su interior señalaba la entrega del dinero en favor del Centro Universitario, ganado gracias a su victoria por una de sus obras más importantes en el repertorio de nuestro personaje, nos referimos al cé- 
lebre cuento: "El Caballero Carmelo". Abraham Valdelomar señaló que, a pesar del sacrificio y tiempo por ayudar a forjar dicha organización, los sanmarquinos no valoraron su esfuerzo, menos aún respaldaron a una persona que podía realizar mejoras en favor de ellos.

Después de esta experiencia, la cual se convirtió en su primer revés electoral, Abraham Valdelomar decidió divorciarse de la política universitaria, emprendiendo el sueño de muchos intelectuales de inicios del siglo XX: viajar a Europa y desarrollar su conocimiento junto con los académicos más importantes del mundo. Su partida al viejo continente fue una oportunidad para reconstruir su imagen, dañada después de su fracaso en las elecciones y su intento de estudiar una carrera universitaria.

\section{Conclusión}

La experiencia universitaria de Valdelomar permite conocer el proceso de transformación social por el cual estaba pasando el Perú al inicio del siglo XX: el ingreso del sector medio a la política nacional. No debe extrañarnos la aparición de instituciones como el Centro Universitario, las cuales buscaban apoyar y reforzar la experiencia en San Marcos de sus jóvenes alumnos, provenientes de la mesocracia nacional.

El intento por convertirse en el máximo dirigente de dicho lugar fue una acción que Valdelomar no la planificó de forma adecuada. Su pésimo resultado académico, su vinculación con el gobierno de Billinghurst, además de una falta de aliados estratégicos al interior de la universidad, propició finalmente su derrota. Si a ello le sumamos que se lo calificó como violento, veremos la razón de la falta de apoyo de la comunidad universitaria. Empero, ello no impidió que años más tarde el Centro Universitario, por el cual pugnó obtener su liderazgo, como institución perdiera protagonismo entre el estudiantado debido a que no cumplió las expectativas de sus miembros. Años después surgirá otra institución que hasta ahora se encuentra vigente en la organización estudiantil: la Federación Universitaria de San Marcos.

\section{Notas}

1 Proposición originaria del Centro Universitario, aprobada y ampliada en Asamblea General (25 de julio de 1908).

2 El Comercio, 9 de abril de 1913.

3 El Comercio, 26 de abril de 1913.

4 El Comercio, 1 de abril de 1913.

5 El Comercio, 4 de mayo de 1913.

6 El Comercio, 9 de mayo de 1913.

7 El Comercio, 4 de mayo de 1912.

8 La Crónica, 5 de mayo de 1913.

9 La Prensa, 18 de mayo de 1913.

10 El Comercio, 11 de mayo de 1913.

11 La Crónica, 9 de mayo de 1913.

12 La Crónica, 17 de mayo de 1913.

13 La Crónica, 20 de mayo de 1913.

14 El Comercio, 23 de mayo de 1912.

15 Loc cit.

\section{Referencias bibliográficas}

Ángeles Caballero, C. (2007). Epistolario de Abraham Valdelomar. Lima: Universidad Alas Peruanas.

Belaunde, V. A. (1967). Trayectoria y destino. Memorias . Lima: Ediventas.

De Priego, M. (2000). Valdelomar. El conde plebeyo. Lima: Fondo Editorial del Congreso del Perú. 
Del Águila, A. (1997). Callejones y mansiones. Espacios de opinión pública y redes sociales y políticas en la Lima del 900. Lima: Pontificia Universidad Católica del Perú.

Deustua, J. y Rénique J. L. (1989). Intelectualidad, indigenismo y descentralismo (19091931). Cusco: Centro de Estudios Regionales Andinos Bartolomé de Las Casas.

Gálvez, J. (1966). Estampas limeñas. Lima: Universidad Nacional Mayor de San Marcos.

Garfias, M. (2009). La formación de la universidad moderna en el Perú (San Marcos, 1850-1919). (Tesis de Licenciatura). Universidad Nacional Mayor de San Marcos, Lima, Perú. https://hdl.handle.net/20.500.12672/2136

González, O. y Paredes, J. (2005). Abraham Valdelomar-Luis Varela Orbegoso: vidas y cartas. Lima: Universidad de San Martín de Porres, Biblioteca Nacional del Perú.

López Soria, J. I. (2007). Valdelomar en la escuela de ingenieros. Lima: Universidad Nacional de Ingeniería.

Memorias. (1908). Memorias de los libros de organización del Centro Universitario. Presentado por el comité organizador. Lima: Imprenta Liberal.

Morales, P. (1912). Juventud. Lima: Imprenta La progresista.

Sánchez, L. A. (1966). Valdelomar ¿mal estudiante? Y un poema inédito. Lima: Editorial Juan Mejía Baca.

Valdelomar, A. (2001a). Los ojos de judas. En Obras Completas, T. I. Lima: Ediciones PetroPerú.

Valdelomar, A. (2001b) Crónica de Lima. En Obras Completas, T. I. Lima: Ediciones PetroPerú.

Valdelomar, A. (2001c). La fiesta de la Luz. El centro universitario y su nuevo local. En Obras Completas, T. II. Lima: Ediciones PetroPerú.

Valdelomar, A. (2001d). La vida universitaria; los exámenes; el centro y los preparativos para el congreso estudiantil. En Obras Completas, T. II. Lima: Ediciones PetroPerú.

Valdelomar, A. (2001e). Notas Universitarias: La presidencia del centro: desistimiento del Sr. Bellido. En Obras Completas, T. Il. Lima: Ediciones PetroPerú. 